\title{
Impact of the resistive wall impedance on beam dynamics in the Future Circular $e^{+} e^{-}$Collider
}

\author{
M. Migliorati* \\ University of Rome 'La Sapienza' and INFN Sez. Romal, 00185 Roma, Italy \\ E. Belli \\ University of Rome 'La Sapienza' and INFN Sez. Romal, 00185 Roma, Italy \\ and CERN, 1217 Meyrin, Geneva, Switzerland \\ M. Zobov \\ INFN-LNF, Frascati, 00044 Roma, Italy
}

(Received 10 January 2018; published 24 April 2018)

\begin{abstract}
The Future Circular Collider study, which aims at designing post-LHC particle accelerator options, is entering in the final stage, which foresees a conceptual design report containing the basic requirements for a hadron and a lepton collider, as well as options for an electron-proton machine. Due to the high beam intensities of these accelerators, collective effects have to be carefully analyzed. Among them, the finite conductivity of the beam vacuum chamber represents a major source of impedance for the electron-positron collider. By using numerical and analytical tools, a parametric study of longitudinal and transverse instabilities caused by the resistive wall is performed in this paper for the case of the Future Circular Collider lepton machine, by taking into account also the effects of coating, used to fight the electron cloud build up. It will be proved that under certain assumptions the coupling impedance of a two layer system does not depend on the conductivity of the coating and this property represents an important characteristic for the choice of the material itself. The results and findings of this study have an impact on the machine design in several aspects. In particular the quite low threshold of single bunch instabilities with respect to the nominal beam current and the not negligible power losses due to the resistive wall are shown, together with the necessity of a new feedback system to counteract the fast transverse coupled bunch instability. The importance of a round vacuum chamber to avoid the quadrupolar tune shift is also discussed. Finally the crucial importance of the beam pipe material coating and thickness choice for the above results is underlined.
\end{abstract}

DOI: 10.1103/PhysRevAccelBeams.21.041001

\section{INTRODUCTION}

The Future Circular Collider (FCC) study [1], which is entering now in its final stage, has the goal of exploring post-LHC particle accelerator options in order to produce a conceptual design report containing the basic requirements for a hadron and a lepton collider, as well as for an electronproton machine. Because of the very high beam currents foreseen in the machines, collective effects have to be analyzed with particular care and attention.

During the study of the single beam collective effects for the lepton collider FCC-ee [2-5], it has been observed that

\footnotetext{
*mauro.migliorati@uniroma1.it
}

Published by the American Physical Society under the terms of the Creative Commons Attribution 4.0 International license. Further distribution of this work must maintain attribution to the author(s) and the published article's title, journal citation, and DOI. the resistive wall impedance, produced by the finite conductivity of the beam vacuum chamber, represents a very important source of impedance and it is responsible of quite low intensity thresholds, for both the microwave instability in the longitudinal plane and the transverse mode coupling instability (TMCI) in the transverse plane, making this contribution very critical for the machine design.

In this paper the impact of the resistive wall impedance in FCC-ee and the consequences on the beam dynamics have been investigated. In Sec. II, it is discussed why this source of impedance is so important for the machine with respect to other contributions and in Sec. III other crucial aspects in the definition of the beam pipe are addressed, as the material coating and thickness. Section IV presents the results of the parametric studies of longitudinal and transverse single bunch beam dynamics, highlighting in particular the low thresholds in both longitudinal and transverse planes, while in Sec. V the multibunch beam dynamics is discussed, by proving the necessity of a novel 
feedback system in the transverse plane, different from the ones used up to now in colliders. Finally, concluding remarks and outlook will end the paper.

\section{THE IMPORTANCE OF RESISTIVE WALL IMPEDANCE IN FCC-ee}

Unlike other machine devices, that in some cases can be optimized from the impedance point of view by using, for example, smooth transitions, high order modes damping systems and novel designs [6], for the resistive wall impedance the possibilities are more limited. Assuming a beam pipe of circular cross section with a single layer of infinite thickness, the exact expressions of the longitudinal and transverse coupling impedances can be found, for example, in Ref. [7]. Under the conditions that

$$
\frac{\chi c}{b} \ll \omega \ll \frac{c \chi^{-1 / 3}}{b}
$$

where $\chi=1 /\left(Z_{0} \sigma_{c} b\right), c$ is the speed of light, $Z_{0}$ is the vacuum impedance, $b$ is the pipe radius and $\sigma_{c}$ is the material conductivity, the exact expression of the impedances can be simplified respectively as

$$
\frac{Z_{||}(\omega)}{C}=\frac{Z_{0} c}{\pi} \frac{1}{[1+i \operatorname{sgn}(\omega)] 2 b c \sqrt{\frac{\sigma_{c} Z_{0} c}{2|\omega|}}}
$$

and

$$
\frac{Z_{\perp}(\omega)}{C}=\frac{Z_{0} c^{2}}{\pi} \frac{2}{[\operatorname{sgn}(\omega)+i] b^{3} c \sqrt{2 \sigma_{c} Z_{0} c|\omega|}}
$$

with $C$ the machine circumference.

It is important to observe that the frequency range of validity of Eqs. (2) and (3), expressed by Eq. (1), is very wide, even if it depends on the vacuum chamber conductivity and radius. By considering, as example, a copper beam pipe with radius of $35 \mathrm{~mm}$, the frequency $f$ must be much greater than $1 \mathrm{~Hz}$ and much lower than $1 \mathrm{THz}$. A bunch length of the order of mm, as that of FCC-ee, fully satisfies these constrains.

It is clear from Eqs. (2) and (3) that the quantities that can be changed to reduce the longitudinal and transverse impedances are only the conductivity of the vacuum chamber (which is made of copper in the case of FCC-ee, as in many other colliders) and the beam pipe radius. Concerning this last parameter, it cannot be increased too much because the power required for the magnets would be too high. A value of $b=35 \mathrm{~mm}$ has been chosen [2], representing a good compromise also for the quadrupole magnets' power. In addition to that, a coating with a material having a conductivity lower than that of copper is required to suppress the electron cloud build up [8-10], and this translates into an increase of the value of the resistive wall impedance.

In order to highlight the importance of the resistive wall impedance in FCC-ee with respect to other colliders, and also as reference for the beam dynamics studies, the main beam parameters used to evaluate the effects of the wake fields [11] are shown in Table I [12].

Consider first the longitudinal plane. Table I shows two important peculiarities of this machine: the low energy spread, necessary to study very narrow particle resonances, and the very low momentum compaction, due to the low dispersion and to the large machine circumference. This combination has a consequence on the microwave instability.

As a first rough approximation, the Boussard criterion [13] can be considered to obtain a scaling of the instability threshold. By using the parameters of the low energy machine with the nominal bunch population, the maximum

\begin{tabular}{|c|c|c|c|c|}
\hline Energy $E_{0}[\mathrm{GeV}]$ & 45.6 & 80 & 120 & 175 \\
\hline Circumference $C[\mathrm{~km}]$ & \multicolumn{4}{|c|}{97.75} \\
\hline Bunch population $N_{p}\left[10^{11}\right]$ & 1.7 & 1.5 & 1.5 & 2.7 \\
\hline Momentum compaction $\alpha_{c}\left[10^{-5}\right]$ & 1.48 & 0.73 & 0.73 & 0.73 \\
\hline Bunch length $\left({ }^{\mathrm{a}}\right) \sigma_{z 0}[\mathrm{~mm}]$ & 3.5 & 3.3 & 3.15 & 2.45 \\
\hline Energy spread $\left({ }^{\mathrm{a}}\right) \sigma_{\varepsilon 0}\left[10^{-4}\right]$ & 3.8 & 6.6 & 9.9 & 14.7 \\
\hline Number of bunches/beam $N_{b}$ & 16640 & 2000 & 393 & 48 \\
\hline Horizontal tune $Q_{x}$ & 269.138 & 389.154 & 389.129 & 389.104 \\
\hline Vertical tune $Q_{v}$ & 269.22 & 391.22 & 391.199 & 391.176 \\
\hline Synchrotron tune $Q_{s}$ & 0.0248 & 0.0229 & 0.0357 & 0.0672 \\
\hline Horizontal emittance $\varepsilon_{x}[\mathrm{~nm}]$ & 0.27 & 0.28 & 0.63 & 1.34 \\
\hline Vertical emittance $\varepsilon_{y}[\mathrm{pm}]$ & 1.0 & 1.0 & 2.3 & 2.7 \\
\hline Longitudinal damping time [ms] & 414 & 77 & 23 & 7.5 \\
\hline SR energy loss/turn $U_{0}[\mathrm{GeV}]$ & 0.036 & 0.34 & 1.72 & 7.8 \\
\hline rf voltage $V_{\text {rf }}[\mathrm{GV}]$ & 0.10 & 0.44 & 2.0 & 9.5 \\
\hline Harmonic number $h$ & \multicolumn{4}{|c|}{130423} \\
\hline
\end{tabular}
allowed longitudinal broad-band impedance $Z_{\|}\left(n \omega_{0}\right) / n$ to

TABLE I. FCC-ee parameter list used to evaluate the beam dynamics effects of wake fields.

${ }^{\mathrm{a}}$ Without beamstrahlung (no collision, worst case). 
avoid the instability, evaluated at the $n$th harmonic of the revolution frequency $\omega_{0}$, is about $0.66 \mathrm{~m} \Omega$, which is quite demanding, even for a very large machine. As a consequence, in order to operate at nominal current below the microwave instability regime, a careful design of the different devices is needed, with the aim of minimizing the impact on the coupling impedance.

This optimization activity is a task which is generally performed in all new accelerators. However, the main difference of FCC-ee with respect to other colliders is the large circumference of $C=97.75 \mathrm{~km}$. Since the resistive wall impedance is proportional to $C$, its contribution to the total broad band impedance does not change with the machine length. This property is not true for other machine devices, i.e., bellows, rf cavities, etc. For example, the number of bellows in an accelerator is not proportional to the machine length: the total number of bellows in KEKB, whose circumference is $3 \mathrm{~km}$, is about 1000 while FCC-ee needs about 8000 bellows, despite a circumference about 30 times larger.

As a consequence, by increasing the machine length, the contribution of the resistive wall impedance with respect to the other devices assumes more importance, becoming critical for the microwave instability, as we will see in Sec. IV.

In the transverse plane, the situation is not as critical as in the longitudinal plane, but presents anyway a low single bunch instability threshold. If we consider the zero azimuthal bunch mode tune shift [14], when this is equal to minus the synchrotron tune, the azimuthal mode zero couples with mode -1 , so that we are in the transverse mode coupling instability regime. The maximum allowed effective transverse impedance to avoid this for the lowest machine energy is about $373.6 \mathrm{k} \Omega / \mathrm{m}$. By considering the impedance due to the resistive wall of Sec. IV without coating and a Gaussian bunch of nominal length, the effective transverse impedance is about $200 \mathrm{k} \Omega / \mathrm{m}$, which is only a factor of two below the threshold.

This conclusion, obtained here by using approximate expressions, will be confirmed in Sec. IV with more accurate simulations.

Before concluding this section, it is important to highlight a final remark about the choice of the beam pipe shape. In order to avoid large incoherent betatron tune shift due to the quadrupolar resistive wall wake fields [15], the vacuum chamber geometry was chosen to be round. Indeed, as a rough estimation of the possible effect of an elliptic chamber, let us evaluate the tune shift for a rectangular vacuum chamber with a cross section of $2 b \times 2 d=70 \times 120 \mathrm{~mm}$, given by the expression [16,17]

$$
\frac{d Q_{x, y}}{d I}= \pm\left(\frac{\pi r}{48 Q_{x, y}}\right)\left(\frac{Z_{0}}{E_{0} / e}\right)\left(\frac{R}{b}\right)^{2}\left(\frac{L}{C}\right), \quad r=\frac{1}{2}+\frac{b^{2}}{d^{2}}
$$

where $Q_{x, y}$ is the transverse tune, $R$ the radius of the machine, $b$ and $d$ the half height and half width of the vacuum chamber, $E_{0}$ the nominal energy, $L / C$ the ring fraction with the rectangular chamber (dipoles). If we assume that the dipole vacuum chambers occupy $L / C=$ 0.71 of the ring, we obtain that the tune shift is about $\pm 0.24 I$. As a consequence, during the beam injection and up to the nominal current of about $1.39 \mathrm{~A}$, the tune variation would be as large as about 0.33 . In order to cope with such big tune changes, additional feedback systems would be necessary to keep the tune constant as required by the beam-beam interaction, dynamic aperture considerations and other beam dynamics issues. The choice of a circular pipe allows to avoid this problem.

Actually the shape of the beam pipe is not totally round, but it has two winglets similar to the SuperKEKB ones [18]. They have the function of antechambers and are used for synchrotron radiation absorbers installation and pumping purposes [19].

\section{RESISTIVE WALL IMPEDANCE WITH COATING}

As mentioned in Sec. I, a thin layer of coating is foreseen to reduce the secondary electron yield for electron cloud mitigation and, in some cases, to help the pumping process [20]. In the following studies, two materials with different conductivities and thicknesses will be considered.

The presence of these coating films makes the surface resistance of the beam pipe higher than the one without coating and the resistive wall effect is more pronounced. In addition to that, there could be also an uncertainty on the coating conductivity which may give inaccurate results about the instability thresholds. This section will show that, in the FCC-ee case, the dominant effect comes mainly from the thickness of the coating while it depends only marginally on its conductivity. Therefore, the best way to decrease the RW contribution is to reduce the coating thickness.

In order to evaluate the impedance of a two-layer beam pipe, let us consider a good conductor with $\sigma_{c} \gg \omega \varepsilon$, with $\varepsilon$ the material dielectric constant. If we have, for example, a minimum bunch length of $2.45 \mathrm{~mm}$ corresponding to the highest energy machine, this has a cut-off frequency of about $19.5 \mathrm{GHz}$. For longer bunches, this frequency is lower. Therefore, by assuming that the frequency of interest for the coupling impedance does not exceed $100 \mathrm{GHz}$, the conductivity of the materials should be much bigger than $10 \mathrm{~S} / \mathrm{m}$, condition verified for copper and in general for all the materials usually used in accelerators for this purpose.

In these hypotheses, if we add the condition that $\omega b /(\gamma \beta c) \simeq 8 \times 10^{-4} \ll 1$, where we have used the maximum frequency of $100 \mathrm{GHz}$ and, for the relativistic factors $\beta$ and $\gamma$, the machine with lowest energy, which is equivalent to ignore space charge effects, the longitudinal and transverse impedances of a two-layer circular beam pipe [21] can be written in a simple form as 

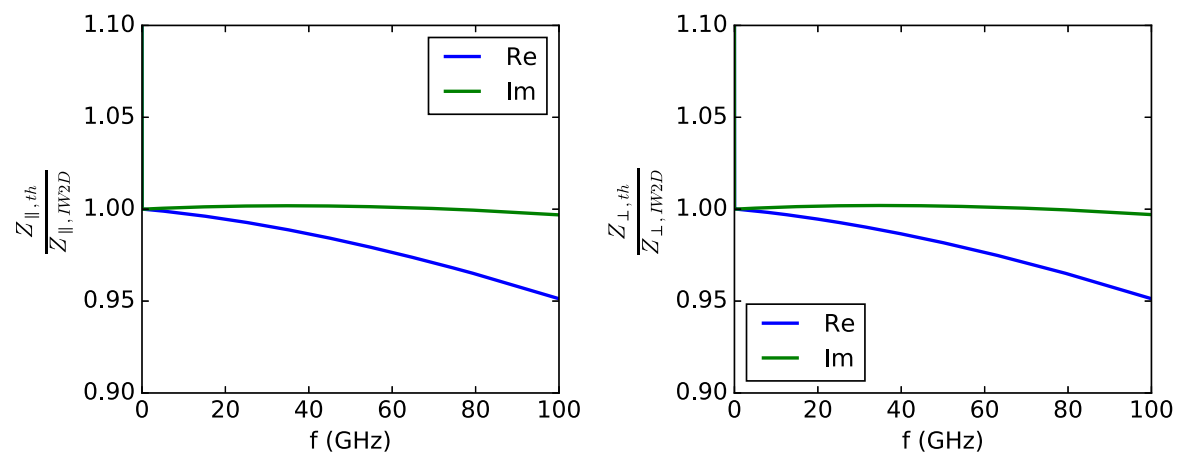

FIG. 1. Ratio of the impedance given by Eq. (8) (left side) and by Eq. (9) (right side) with respect to the results of IMPEDANCEWAKE2D. In blue the real part of the impedance is represented, and in green the imaginary one. A copper pipe of 35 mm of radius with a coating conductivity of $\sigma_{1}=2 \times 10^{6} \mathrm{~S} / \mathrm{m}$ and a thickness of $100 \mathrm{~nm}$ has been used.

$\frac{Z_{\|}(\omega)}{C}=\frac{Z_{0} \omega}{4 \pi c b}\left\{[\operatorname{sgn}(\omega)-i] \delta_{1} \frac{\alpha \tanh \left[\frac{1-i \operatorname{sgn}(\omega)}{\delta_{1}} \Delta\right]+1}{\alpha+\tanh \left[\frac{1-i \operatorname{sgn}(\omega)}{\delta_{1}} \Delta\right]}\right\}$,

and

$\frac{Z_{\perp}(\omega)}{C}=\frac{Z_{0}}{2 \pi b^{3}}[1-i \operatorname{sgn}(\omega)] \delta_{1} \frac{\alpha \tanh \left[\frac{1-i \operatorname{sgn}(\omega)}{\delta_{1}} \Delta\right]+1}{\alpha+\tanh \left[\frac{1-i \operatorname{sgn}(\omega)}{\delta_{1}} \Delta\right]}$,

where

$$
\delta_{1}=\sqrt{\frac{2 c}{\omega \sigma_{c 1} Z_{0}}}
$$

is the skin depth of the coating, $\Delta$ the corresponding thickness, and, for a good conductor, $\alpha \simeq \delta_{1} / \delta_{2}$, with $\delta_{2}$ the skin depth of the substrate, which is supposed to be of infinite thickness.

If we now consider the important assumption that the skin depth of the coating material is much bigger than its thickness, that is $\delta_{1} \gg \Delta$, then the longitudinal and transverse impedances can be written as

$$
\begin{aligned}
\frac{Z_{\|}(\omega)}{C} & =\frac{Z_{0} \omega}{4 \pi c b} \frac{[\operatorname{sgn}(\omega)-i] \delta_{2}-2 i \Delta}{1+\frac{\delta_{2} \Delta[1-i \operatorname{sgn}(\omega)]}{\delta_{1}^{2}}} \\
& \simeq \frac{Z_{0} \omega}{4 \pi c b}\left\{[\operatorname{sgn}(\omega)-i] \delta_{2}-2 i \Delta\left(1-\frac{\sigma_{1}}{\sigma_{2}}\right)\right\},
\end{aligned}
$$

and

$$
\begin{aligned}
\frac{Z_{\perp}(\omega)}{C} & =\frac{Z_{0}}{2 \pi b^{3}}[1-i \operatorname{sgn}(\omega)] \delta_{1} \frac{[1-i \operatorname{sgn}(\omega)] \Delta+\delta_{2}}{1+\frac{\delta_{2} \Delta[1-i \operatorname{sgn}(\omega)]}{\delta_{1}^{2}}} \\
& \simeq \frac{Z_{0}}{2 \pi b^{3}}\left\{[1-i \operatorname{sgn}(\omega)] \delta_{2}-2 i \Delta \operatorname{sgn}(\omega)\left(1-\frac{\sigma_{1}}{\sigma_{2}}\right)\right\},
\end{aligned}
$$

where $\sigma_{1}$ is the coating conductivity and $\sigma_{2}$ the substrate one, and, in the last passage, we have ignored second order terms in the ratio $\Delta / \delta_{1}$.

In order to demonstrate that all the assumptions we made to derive these two expressions are valid in our case, in Fig. 1, we show the ratio in the longitudinal and transverse case of the above simple approximated expressions with respect to the exact solution obtained with the code IMPEDANCEWAKE2D [22] for a copper pipe of $35 \mathrm{~mm}$ of radius with a coating having a conductivity of $2 \times 10^{6} \mathrm{~S} / \mathrm{m}$ (corresponding, as example, to TiN material) and a thickness of $100 \mathrm{~nm}$. With these parameters the ratio $\Delta / \delta_{1}$ is about 0.09 at the highest frequency of $100 \mathrm{GHz}$ (with the smallest skin depth).

As we can see from the figure, in the worst case at $100 \mathrm{GHz}$, the difference in the impedances is only of about $5 \%$ for the real part, while the imaginary part is essentially the same, indicating that the two expressions (8) and (9) can be used with very good approximation to evaluate the impedance of the two-layer beam pipe when the coating thickness is much lower than its skin depth.

Equations (8) and (9) are also important for another reason. The first term in the curly brackets on the right side of the equations represents actually the impedance of a single layer given by Eqs. (2) and (3). As a consequence, we can read the longitudinal and transverse impedance of the two-layer beam pipe as that given by the single layer plus an inductive perturbation proportional to the thickness. The same conclusion has also been obtained in Ref. [23]. In addition to that, if $\sigma_{1} / \sigma_{2} \ll 1$, which is generally verified for any of the coating materials used for suppressing the electron cloud build up, then we can ignore this ratio with respect to 1 and the perturbation becomes independent on the coating conductivity.

This is also a very important result, indicating that if we manage to reduce the coating to a very low value such that, up to the maximum frequency of interest, the skin depth remains much higher than its thickness, then we can ignore the material conductivity, and we simply add a term to the imaginary part of the single layer impedance proportional 

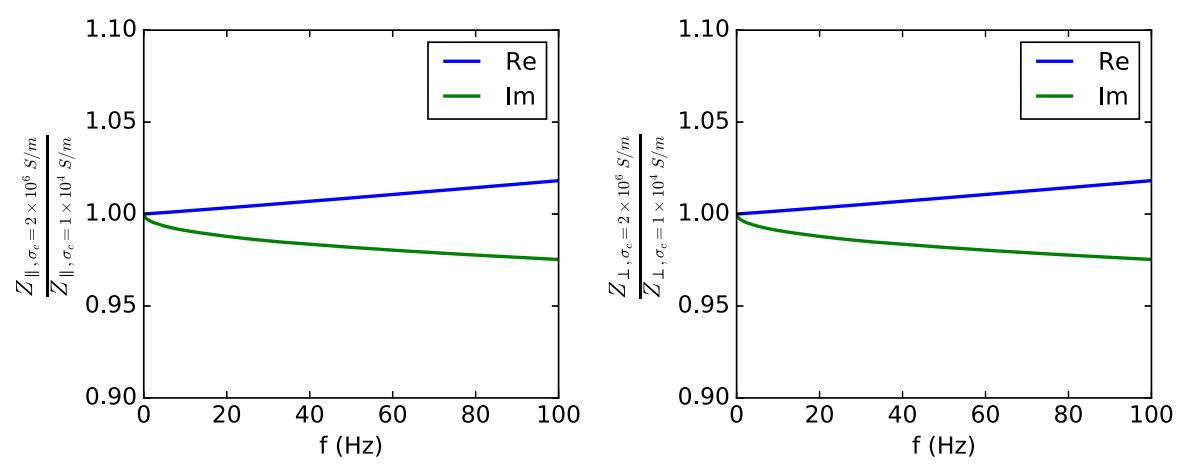

FIG. 2. Ratio of the impedance of two-layer beam pipe with coating conductivities of $2 \times 10^{6} \mathrm{~S} / \mathrm{m}$ and $10^{4} \mathrm{~S} / \mathrm{m}$ as given by IMPEDANCEWAKE2D. A copper pipe of $35 \mathrm{~mm}$ of radius with a coating thickness of $100 \mathrm{~nm}$ has been used. On the left side the longitudinal impedance is shown, with the real part in blue and the imaginary one in green, and on the right side the transverse one.

to the thickness itself. Moreover, the real part of the impedance is not affected by the coating and then beam losses remain the same as those without coating. As example of this conclusion, in Fig. 2 we show the ratio of the impedance for a two-layer beam pipe having a coating with conductivity of $2 \times 10^{6} \mathrm{~S} / \mathrm{m}$ with respect to that with $10^{4} \mathrm{~S} / \mathrm{m}$, which is more than two order of magnitudes lower. The longitudinal and transverse impedances are shown on the left and right side, respectively. The coatings have a thickness of $100 \mathrm{~nm}$, and the results have been obtained without any approximation with the exact solution of the code IMPEDANCEWAKE2D.

We can see from the figure that in this large variation of conductivities, as long as the thickness of the coating remains much smaller that the skin depths, with a value, for example, of $100 \mathrm{~nm}$, the impedance is essentially the same, with a small difference of about $2 \%$ up to a frequency of $100 \mathrm{GHz}$. If we increase the thickness, at the beginning the perturbation to the imaginary part of the impedance increases linearly, up to when the thickness becomes comparable with the skin depth, after which the expression becomes more complicated.

Considering that the resistive wall effect is so important for the FCC-ee machine, we can conclude that the thickness of the coating can play a very crucial role, and the situation can be very challenging unless we reduce this quantity. If it is thinner than the skin depth, even if it is not invisible, however it gives a pure inductive contribution proportional to the thickness and independent on its conductivity.

This very important result indicates that, in order to reduce the resistive wall impedance in presence of a coating, we can move in the direction of small thickness without paying much care to the material conductivity. As a consequence of this, investigations about the coatings are focusing on the minimum achievable thickness that could be reached with Non Evaporable Getters (NEG) (which is the preferred material for FCC-ee due also to its pumping capacity) without compromising its properties as means to fight the electron cloud build up and pumping capacity.

\section{SINGLE BUNCH BEAM DYNAMICS}

In Sec. II, the impact of the resistive wall impedance on the single bunch beam dynamics has been evaluated by using analytical approximated expressions. Here a more rigorous numerical study is performed with the help of the macroparticle tracking code PyHEADTAIL [24]. Some of the results have been benchmarked against other simulation codes which take into account collective effects [2527]. For these studies, the vacuum chamber is assumed to be circular with $35 \mathrm{~mm}$ radius and three layers: a first layer of copper with $2 \mathrm{~mm}$ thickness, then $6 \mathrm{~mm}$ of dielectric and finally iron with resistivity $\rho=10^{-7} \Omega \mathrm{m}$. It is also assumed to be coated with two different materials that in the following will be referred to as material $\mathrm{A}$ and material B with conductivities $\sigma_{c, A}=10^{4} \mathrm{~S} / \mathrm{m}$ and $\sigma_{c, B}=2 \times 10^{6} \mathrm{~S} / \mathrm{m}$, respectively. In order to study the effects of the thickness of these coatings on the single bunch beam dynamics, four different thicknesses will be taken into account: $1 \mu \mathrm{m}, 200 \mathrm{~nm}, 100 \mathrm{~nm}$ and $50 \mathrm{~nm}$.

Figure 3 shows the real and imaginary part of the transverse and longitudinal RW impedances obtained by the IMPEDANCEWAKE2D code as a function of the frequency for material B with all the thicknesses under study. For the transverse impedance, the logarithmic scales have been used to highlight the behaviour at low frequency, which is important for the study of the transverse coupled bunch instabilities, as we will see in Sec. VA.

\section{A. Longitudinal dynamics}

As mentioned above, the numerical simulations have been performed with the PYHEADTAIL code. As most of the macroparticle tracking codes, PYHEADTAIL computes the wake potential as the convolution between the longitudinal wake function $w_{\|}(z)$, i.e., the Green function of a point charge, and the bunch distribution $\lambda(z)$ :

$$
W_{\|}(z)=\int_{-\infty}^{z} w_{\|}\left(z-z^{\prime}\right) \lambda\left(z^{\prime}\right) d z^{\prime}
$$



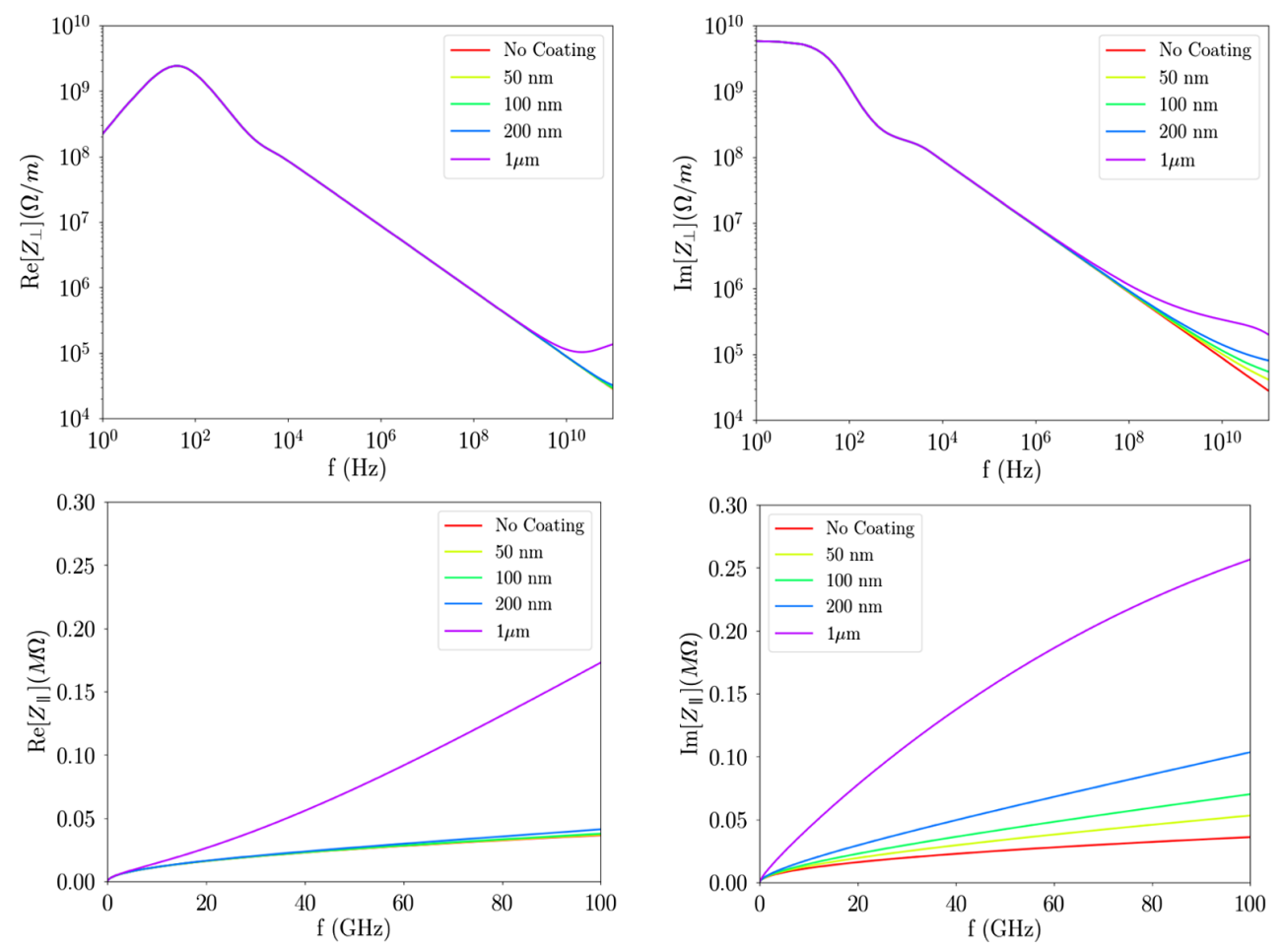

FIG. 3. Real and imaginary part of transverse (top) and longitudinal (bottom) RW impedances as a function of the frequency in the case of no coating, with a copper pipe of $35 \mathrm{~mm}$ of radius, and with different thicknesses of coating $\mathrm{B}$.

This integral represents the energy gained or lost by a charge due to the entire bunch and it has to be evaluated at each turn for each macroparticle. In order to have reliable results, a simulation usually tracks $10^{6}$ macroparticles, which translates into more than $10^{11}$ operations at each turn for the computation of the wake potential. In order to reduce the number of operations, the bunch is usually divided into slices, each one containing a certain number of macroparticles and assumed to act as a point charge. The wake potential is then computed at the center of each slice and interpolated for all the macroparticles of the bunch. However, the input wake function can cause some problems to simulations based on this approach, since it varies in very short distances and a very large number of slices would be necessary, thus increasing the computational time. One possible solution is to use, as Green function for the code, the wake potential of a very short bunch, i.e., the wake potential of a bunch with a bunch length $\sigma_{z} \simeq \frac{1}{10} \sigma_{z \text {,nominal }}$. Figure 4 shows the analytic wake potentials for a Gaussian bunch with nominal bunch length $\sigma_{z \text {,nominal }}=3.5 \mathrm{~mm}$ compared to the wake potentials obtained from PyHEADTAIL as solutions of the convolution integral of Eq. (10), in the case of no coating and different thicknesses of coating B. Results reported in Fig. 4 show that the wake potential of a very short bunch can be treated as the wake potential generated from of a point charge, for all the cases under study.

\section{Potential well distortion, bunch lengthening and microwave threshold}

One important effect of the longitudinal wakefield on the single bunch dynamics is the distortion of the bunch shape. The shape of a bunch is Gaussian at low intensities while there is a distortion as the bunch intensity increases. For intensities below the so-called microwave instability

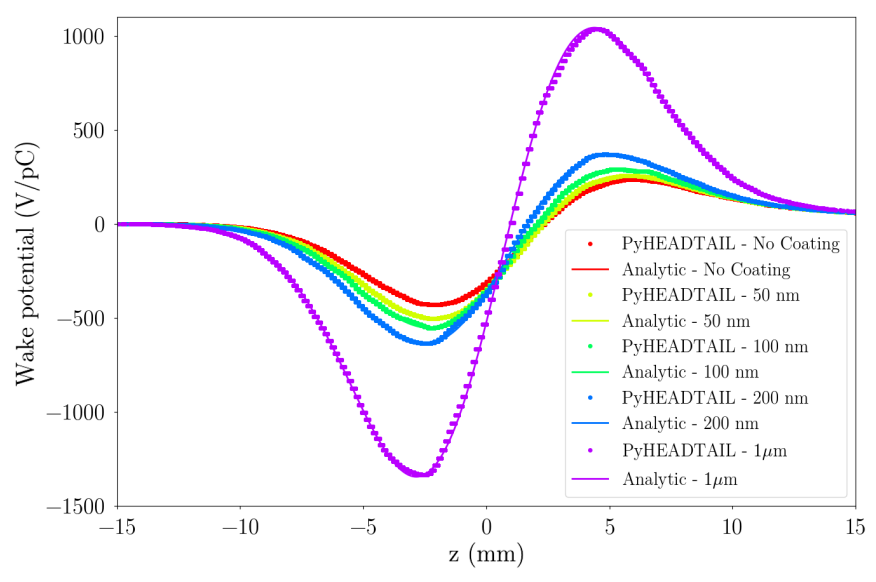

FIG. 4. Comparison of the analytical longitudinal wake potentials of a $3.5 \mathrm{~mm}$ Gaussian bunch with the wake potentials obtained from PYHEADTAIL as convolution between the longitudinal bunch distribution and the wake potential of a $0.35 \mathrm{~mm}$ Gaussian bunch in the case of no coating with a copper pipe of $35 \mathrm{~mm}$ of radius, and with different thicknesses of coating $\mathrm{B}$. 


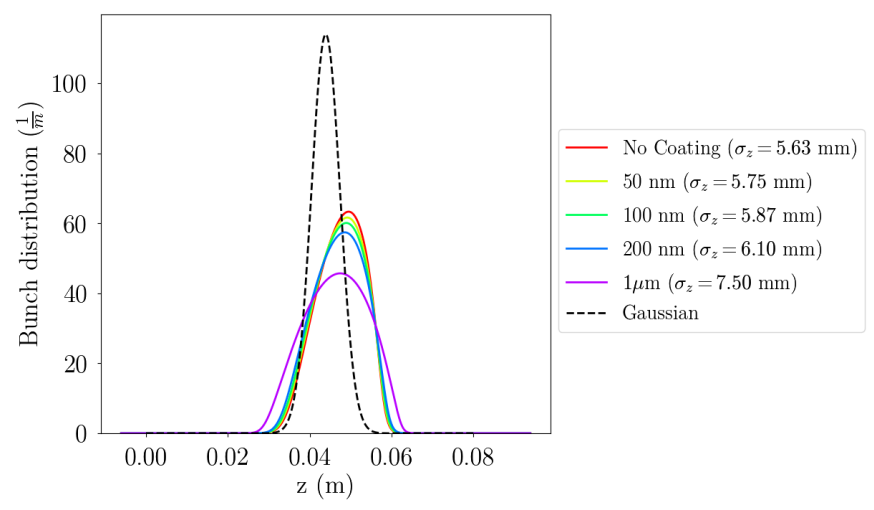

FIG. 5. Longitudinal bunch distribution obtained from Haissinski equation at nominal intensity in the case of no coating and different thicknesses of coating B. The dashed black line represents the Gaussian equilibrium shape. The other machine parameters are those of Table I with the lowest machine energy.

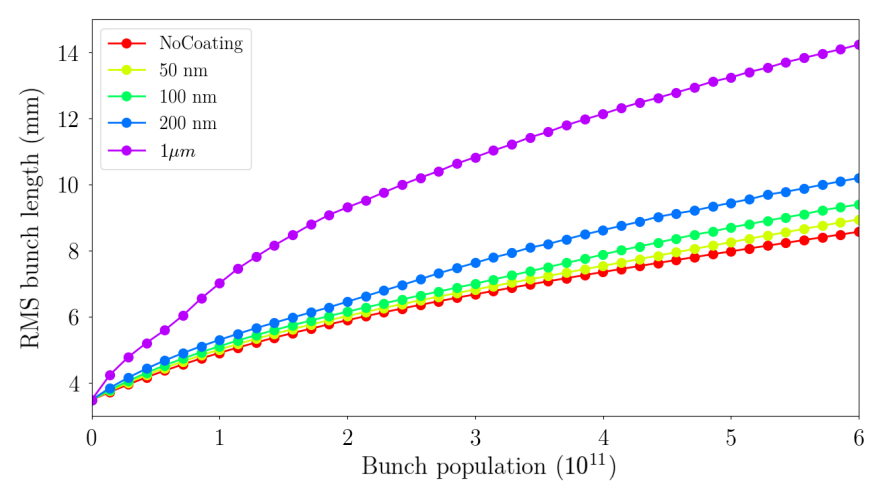

FIG. 6. Bunch length as a function of the bunch intensity obtained from numerical simulations in the case of no coating and with different thicknesses of coating B. The other machine parameters are those of Table I with the lowest machine energy.

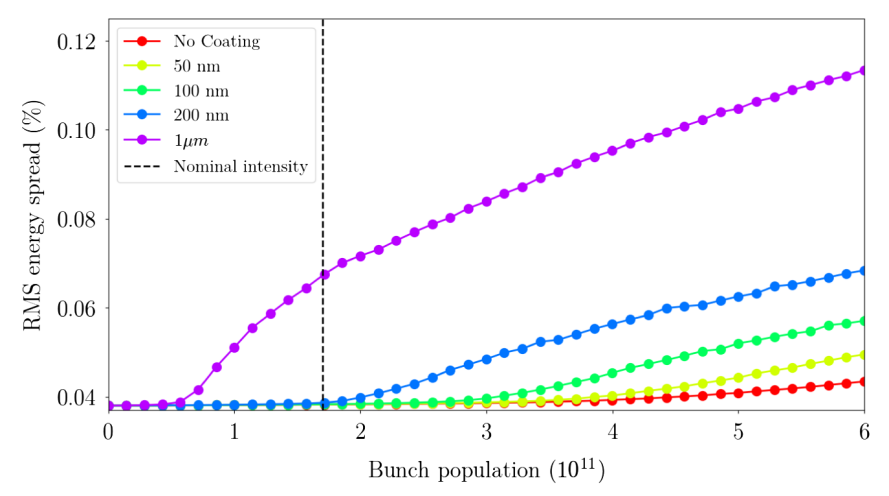

threshold, the bunch shape can be predicted with the Haissinski integral equation in the form [28]

$$
\lambda(z)=\lambda_{0} e^{\frac{1}{E_{0} \eta \sigma_{\epsilon_{0}}^{2}} \Psi(z)}
$$

with

$$
\begin{aligned}
\Psi(z)= & \frac{1}{C}\left[\int_{0}^{z}\left[e V_{\mathrm{rf}}\left(z^{\prime}\right)-U_{0}\right] d z^{\prime}\right. \\
& \left.-e^{2} N_{p} \int_{0}^{z} d z^{\prime} \int_{-\infty}^{z^{\prime}} \lambda\left(z^{\prime \prime}\right) \omega_{\|}\left(z^{\prime}-z^{\prime \prime}\right) d z^{\prime \prime}\right]
\end{aligned}
$$

where $\lambda_{0}$ is a constant determined by normalization, $\eta$ is the slippage factor, $\sigma_{\varepsilon 0}$ is the rms energy spread, $V_{\mathrm{rf}}$ is the $\mathrm{rf}$ peak voltage, $U_{0}$ is the energy lost per turn due to synchrotron radiation, and $N_{p}$ the bunch population.

Figure 5 shows the bunch shape at the nominal bunch intensity of $1.7 \times 10^{11}$ with the corresponding bunch length in the case of no coating and with different thicknesses of coating $\mathrm{B}$.

In the case of the RW impedance, the Haissinski equation predicts an increase of the bunch length with the bunch intensity which finds a good agreement with the one predicted by numerical simulations, as shown in Fig. 6, except for the $1 \mu \mathrm{m}$ thickness case that is therefore expected to be longitudinally unstable.

On the other hand, the energy spread remains constant below the instability threshold, above which it starts to increase with the bunch intensity. Figure 7 shows the energy spread obtained from simulations as a function of the bunch population for all the cases under study. As example, the plot on the left refers to material B only, showing that for the same conductivity a smaller thickness allows to reduce significantly the microwave instability threshold. In this particular case, a coating of $1 \mu \mathrm{m}$ thickness makes the bunch unstable (as predicted before) while the instability threshold is increased of about a factor 7 in the case of the thinnest film of $50 \mathrm{~nm}$. The plot on the right

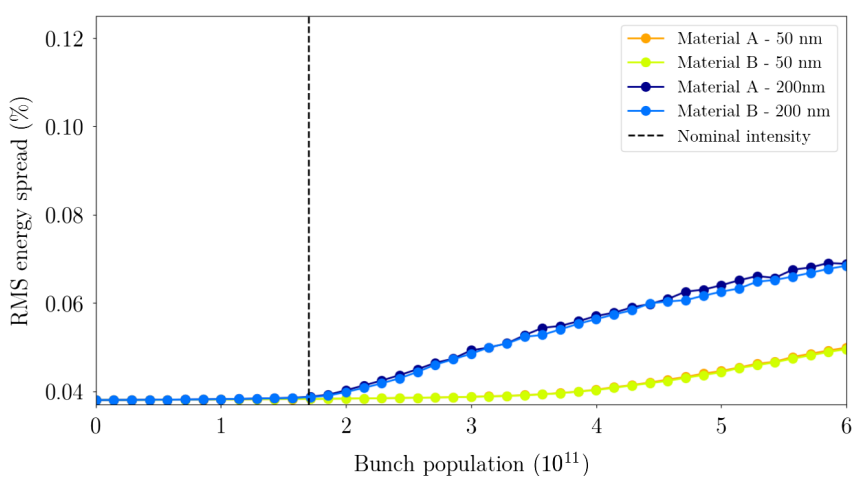

FIG. 7. Energy spread as a function of the bunch intensity obtained from numerical simulations in the case of no coating and with different thicknesses of coating B (left side) and for both materials A and B in the two cases of 50 and 200 nm thickness (right side). The other machine parameters are those of Table I with the lowest machine energy. The dashed black line corresponds to the nominal bunch population. 
considers both materials $\mathrm{A}$ and $\mathrm{B}$, pointing out that the material conductivity does not affect the microwave threshold for small thicknesses, as discussed in Sec. III.

\section{Loss factor}

When the bunch interacts with the wakefield, it loses some energy that has to be compensated by the rf system every turn with a synchronous phase shift:

$$
U_{\mathrm{WF}}=-\Delta \phi e V_{\mathrm{rf}} \cos \left(\phi_{s}\right)
$$

where $\Delta \phi$ is the phase shift for a given bunch intensity and $\phi_{s}$ is the synchronous phase. Since the energy lost per turn is related to the loss factor by the following formula:

$$
U_{\mathrm{WF}}=e^{2} N_{p} k_{\|}
$$

one obtains

$$
k_{\|}=-\frac{\Delta \phi V_{\mathrm{rf}} \cos \left(\phi_{s}\right)}{e N_{p}}
$$

Figure 8 shows the loss factor as a function of the bunch length obtained by using Eq. (15) with $\Delta \phi$ given by numerical simulations' data, in the case of no coating (red curve) and $50 \mathrm{~nm}$ thickness for both materials A and B (blue and yellow curves, respectively). The black curve corresponds to the analytic loss factor of a Gaussian bunch in a copper beam pipe with conductivity $\sigma_{c} \simeq 6 \times 10^{7} \mathrm{~S} / \mathrm{m}$, given by the formula [29]

$$
\frac{k_{\|}}{L}=\frac{\Gamma\left(\frac{3}{4}\right) c}{4 \pi^{2} b \sigma_{z}^{\frac{3}{2}}} \sqrt{\frac{Z_{0}}{2 \sigma_{c}}}\left[\frac{V}{C}\right]
$$

with $\Gamma\left(\frac{3}{4}\right)=1.225$.

The small difference between the results of Eq. (16) and the numerical simulation, of the order of about $4 \%$ for $50 \mathrm{~nm}$ of coating, for example, is mainly due to the bunch shape difference which, for the simulations, is distorted from Gaussian according to Eq. (12). It is important to highlight that, for such a small coating thickness, the loss factor does not depend on the coating conductivity and it is equal to the one without coating, thus confirming the results of Sec. III, where we have shown that the contribution to the impedance of the coating is only inductive for sufficiently small thicknesses.

From the loss factor it is possible to derive the total power lost per turn by the beam as

$$
P=\frac{N_{b}\left(N_{p} e\right)^{2} k_{\|}}{T_{0}}
$$

with $N_{b}$ the number of bunches per beam and $T_{0}$ the revolution period. From Fig. 8, the loss factor at nominal intensity is about $100 \mathrm{~V} / \mathrm{pC}$ and it is almost independent on the presence of the coating and on the material

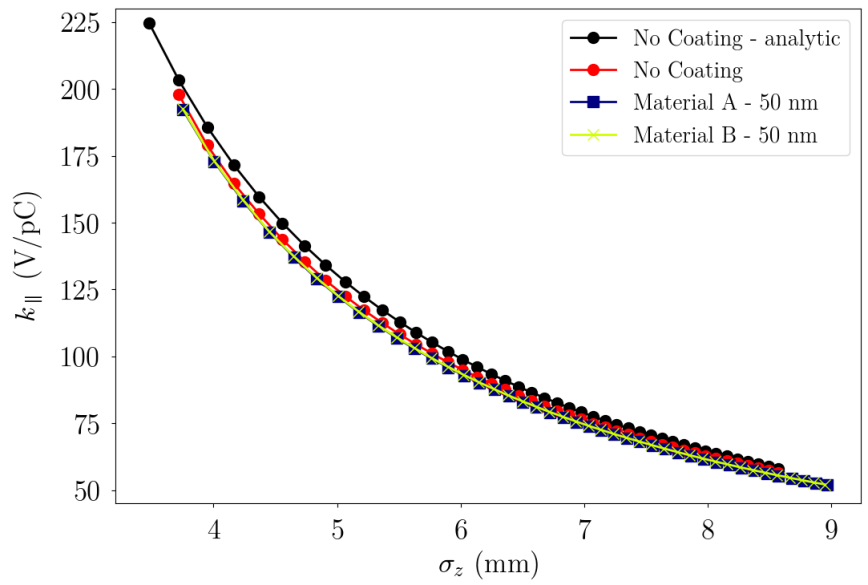

FIG. 8. Loss factor as a function of the bunch length obtained from analytic formula in the case of no coating (black curve) compared with numerical simulations in the case of no coating and $50 \mathrm{~nm}$ thickness for both materials $\mathrm{A}$ and $\mathrm{B}$. The other machine parameters used for the numerical simulations are those of Table I with the lowest machine energy.

conductivity. This value, only due to the resistive wall, corresponds to a total power lost per beam of about $3.8 \mathrm{MW}$ at $\mathrm{Z}$ running, representing about $10 \%$ of the synchrotron radiation power of $50 \mathrm{MW}$ dissipated by the beam.

\section{Incoherent synchrotron frequency shift and spread}

Since the wake potential oscillates rigidly with the bunch, the wakefield does not affect the dipole (rigid) coherent synchrotron frequency, which remains unchanged. However all the other higher coherent modes (quadrupolar, sextupolar etc.) are affected because they do not represent rigid bunch motions. In addition, the internal motion of the particles in the bunch is also affected, thus producing an incoherent synchronous frequency shift relative to the coherent frequency that could lead to the loss of Landau damping [30], if comparable or larger than the incoherent frequency spread. Even if, for electron machines, the effect may be not very dangerous thanks to the natural damping mechanism due to the synchrotron radiation, a too high incoherent frequency shift can affect, for example, the beam-beam performance.

In order to evaluate the effect of the wakefield on the incoherent tune spread, the synchrotron frequency of the macro-particles at the nominal bunch intensity has been obtained with PYHEADTAIL. In Fig. 9, an histogram of the frequency distribution with and without wakefield is shown, indicating a quite high incoherent tune shift of about a factor 2 with respect to the unperturbed synchrotron tune of 0.0248 . A coating of $100 \mathrm{~nm}$ has been used for the simulations.

Figure 9 also shows a large incoherent spread, comparable with the synchrotron tune value. Therefore, it is worthwhile to investigate how the incoherent synchrotron 


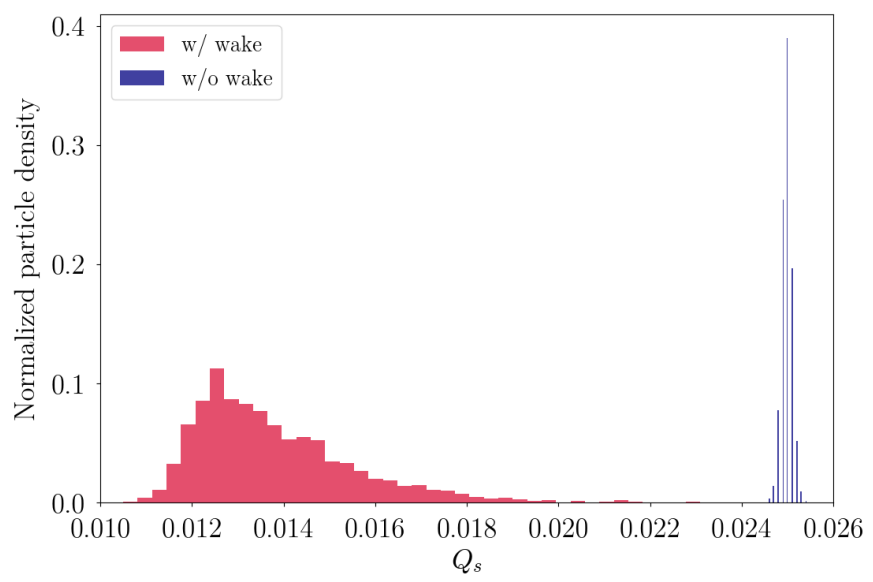

FIG. 9. Incoherent synchrotron tune distribution at nominal intensity, produced by the resistive wall impedance with a coating of $100 \mathrm{~nm}$. The results are obtained with PYHEATAIL by using the machine parameters of Table I at the lowest machine energy.

tune spread affects the recently discovered X-Z coherent beam-beam instability appearing in collisions with a large angle and coupling the horizontal and longitudinal motion [31]. Namely this coherent instability is important for the parameter choice of the FCC-ee [32].

\section{B. Transverse dynamics}

One important effect of the transverse short range RW wakefield is the TMCI: the betatron frequencies of the intrabunch modes shift when the bunch intensity increases and an instability occurs when the coherent frequencies of two modes merge together. Unlike the microwave instability in the longitudinal case, above the transverse instability threshold the bunch is lost and this makes the TMCI very dangerous for the beam.

As already pointed out in Sec. II, we expect an important RW effect also in the transverse plane. In Fig. 10, the real part of the frequency (tune shift) of the first two radial coherent oscillation modes, with the azimuthal number going from -2 to 2 , as a function of the bunch population is shown. The shifts have been obtained with DELPHI code [33], which uses a decomposition over orthogonal Laguerre polynomials for the radial modes, and by considering the transverse impedance of material B with $50 \mathrm{~nm}$ (left side) and $1 \mu \mathrm{m}$ (right side) of coating thickness.

For each point in the plots, the bunch length at a given intensity, as obtained by PYHEADTAIL, has been used. The instability threshold is about a factor of 2 higher than the nominal bunch population. It is important to note that also in this case the coating thickness affects the threshold, according to the results of Sec. III. However, the difference is not so high between $50 \mathrm{~nm}$ and $1 \mu \mathrm{m}$ because, for this last case, a higher bunch length at the same intensity helps to increase the transverse threshold. In addition, the results with the conductivity of material A are the same of those of Fig. 10, confirming that for small thickness the impedance is almost independent on the coating conductivity.

Finally, another important effect related to TMCI, is due to the collision with the other beam: two counterrotating beams can produce coherent dipole oscillation modes modifying the stability conditions. In this situation, coherent beam-beam effects and impedance are not independent any more and their interplay should be taken into account [34]. This subject, along with the mitigating effect of beamstrahlung, equally linked to the colliding beams, is an important topic, which deserves future investigations.

\section{MULTIBUNCH DYNAMICS}

\section{A. Transverse dynamics}

For the multibunch case, the most critical situation is related to the transverse coupled bunch instability due to the long range RW wakefield. If we consider the motion of

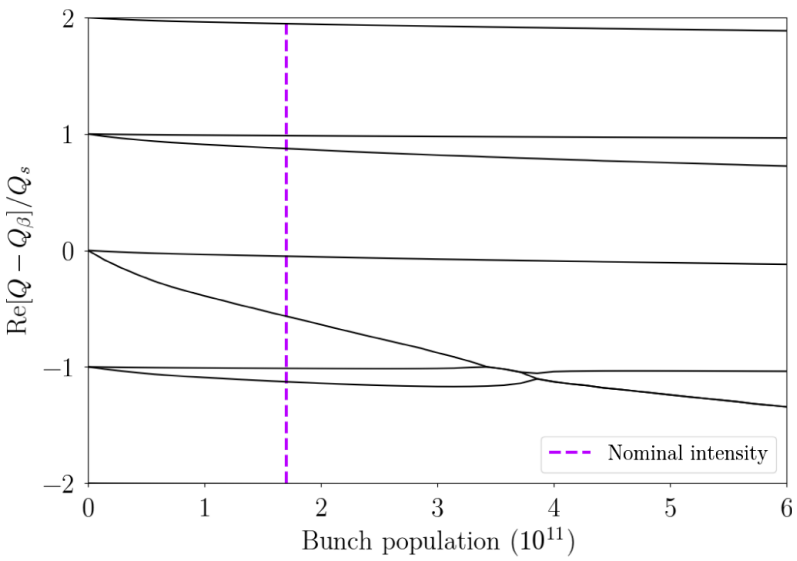

FIG. 10. Real part of the frequency of the first coherent oscillation modes as a function of bunch population with $50 \mathrm{~nm}$ (left side) and the $1 \mu \mathrm{m}$ of coating thickness for material B (right side). The curves are obtained with DELPHI code by using the machine parameters of Table I at the lowest machine energy and with the bunch length given by PYHEADTAIL at different intensities. The dashed vertical lines correspond to the nominal bunch population. 

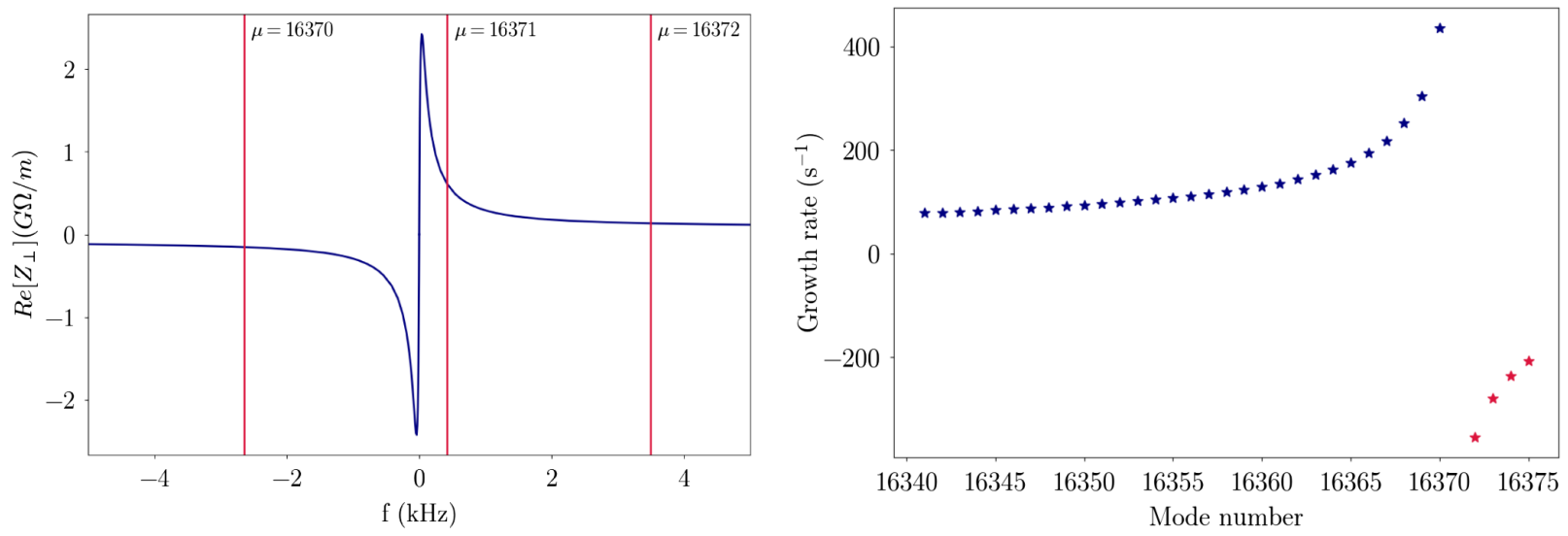

FIG. 11. Vertical coupled bunch spectrum, obtained by using the machine parameters of Table I at the lowest energy, and real part of the resistive wall impedance for a copper beam pipe of $35 \mathrm{~mm}$ without coating, as a function of frequency around $f=0$ (left side), and growth rate of the coupled bunch modes with azimuthal mode number $m=0$ (right side).

a beam as sum of coherent coupled bunch modes, the growth rate of the lowest azimuthal mode $m=0$ for a Gaussian bunch is given by [35]

$$
\alpha_{\mu, \perp}=-\frac{c I}{4 \pi\left(E_{0} / e\right) Q_{\perp}} \sum_{q=-\infty}^{\infty} \operatorname{Re}\left[Z_{\perp}\left(\omega_{q}\right)\right]
$$

where we assumed the form factor due to the bunch shape [2] to be about 1 and

$$
\omega_{q}=\omega_{0}\left(q N_{b}+\mu+Q_{\perp}\right)
$$

Each $\mu$ is an integer number from 0 to $N_{b}-1$ representing a coupled bunch mode. The instability happens when $\alpha_{\mu}$ is positive, i.e., when the impedance is evaluated at negative frequencies. By considering the most dangerous mode, which is the one with the coherent frequency $\omega_{q}$ closest to zero and negative, and by using as approximation a single betatron frequency line instead of the sum over q, for the vertical plane with a fractional part of the tune of 0.22 the growth rate given by Eq. (18) is about $435 \mathrm{~s}^{-1}$, corresponding to about 7 turns. These values have been obtained by using $100 \mathrm{~nm}$ of coating with material $\mathrm{B}$, and the results do not change significantly for other thicknesses and/or materials, since the real part of the impedance is not affected by these parameters, as already discussed in Sec. III. It is worthwhile to note that the transverse radiation damping time is about $0.83 \mathrm{~s}$, corresponding to 2550 turns, i.e., it is much longer than required for the instability suppression.

Figure 11 (left side) shows the beam spectrum of three coupled bunch modes and the real part of the transverse impedance without coating and close to zero frequency, while the right plot shows the growth rate as a function of the coupled bunch mode number, highlighting the presence of several unstable modes that need to be damped.

The rise times of these modes are in the range of few milliseconds, which, in other lepton factories, are commonly cured by transverse bunch by bunch feedback systems [36]. However, due to the large circumference of
FCC, here the rise times correspond to few turns, making necessary the realization of a new challenging feedback system. Some schemes that could deal with this problem have been proposed in Ref. [37].

\section{CONCLUSIONS}

In this paper we have shown that for FCC-ee the resistive wall beam coupling impedance plays a crucial role affecting both the parameters choice and some design solutions of the $100-\mathrm{km}$ collider. In particular, it has been proposed to use the vacuum chamber with a round cross section almost everywhere in the rings in order to avoid the strong betatron tune shift with beam current due to quadrupolar resistive wall wake fields.

The vacuum chamber coatings used to mitigate the electron cloud effects and to improve the pumping speed are shown to increase drastically the machine impedance. It has been demonstrated analytically and confirmed numerically that for the considered bunch lengths the RW impedance of a coated vacuum chamber mainly depends on the coating thickness and only marginally on the coating conductivity: a thin film of the order of 50-100 nm is accepted to keep the bunch nominal intensity under the single bunch microwave instability threshold. In order to understand if such thin coatings work well for suppression of electron cloud effects and for improvement of pumping speed, we have started a campaign of measurements using NEG with different thicknesses. This will be the subject of a future work. In addition, a large momentum compaction factor would be preferable to increase the instability threshold.

The TMCI instability threshold due to the RW is a bit less stringent, but still dangerous. It is by about a factor 2 higher than the nominal bunch intensity. However, even in this case, a coating thickness as small as possible is required in order to have larger margins keeping in mind the geometric impedance contributions of other vacuum chamber components. 
The power loss due to the RW impedance is also not negligible. It is estimated to be of the order of $10 \%$ of the total losses due to the synchrotron radiation. As a consequence, additional rf cavities are necessary to compensate the RW losses. In addition, the beam interaction with the vacuum chamber resistive walls results in the synchrotron tune reduction and a large incoherent synchrotron tune spread. Both effects can influence the beam-beam performance shifting the collider working point and affecting the incoherent beam-beam resonances.

The transverse coupled bunch instability driven by the resistive wall impedance is very fast. Its rise time is evaluated to be a few revolution turns, thus requiring a robust and novel feedback system to suppress the instability.

Finally, due to its importance, the RW impedance can be considered as the reference impedance at the design stage and it can be used for comparison with all other geometric impedance contributions.

\section{ACKNOWLEDGMENTS}

We acknowledge many helpful and stimulating discussions with E. Métral. This work was supported in part by the European Commission under the HORIZON2020 Integrating Activity project ARIES, Grant agreement No. 730871, and in part by the MICA project of INFN.

[1] https://fcc.web.cern.ch/.

[2] E. Belli, M. Migliorati, S. Persichelli, and M. Zobov, Single beam collective effects in FCC-ee due to beam coupling impedance, arXiv:1609.03495.

[3] M. Migliorati, E. Belli, G. Castorina, S. Persichelli, B. Spataro, and M. Zobov, Collective effects issues for FCCee, in Proceedings of eeFACT2016, Cockcroft Institute, Daresbury, UK (2016), p. 100, TUT3AH1, http://accelconf .web.cern.ch/AccelConf/eefact2016/papers/tut3ah1.pdf.

[4] E. Belli, M. Migliorati, and G. Rumolo, Electron cloud and collective effects in the interaction region of FCC-ee, in Proceedings of eeFACT2016, Cockcroft Institute, Daresbury, UK (2016), p. 130, TUT3AH7, http://accelconf.web .cern.ch/AccelConf/eefact2016/papers/tut3ah7.pdf.

[5] E. Belli, G. Castorina, M. Migliorati, A. Novokhatski, S. Persichelli, B. Spataro, and M. Zobov, Coupling impedances and collective effects for FCC-ee, in 8th International Particle Accelerator Conference (IPAC'17), Copenhagen, Denmark (2017), p. 3734, http://accelconf .web.cern.ch/AccelConf/ipac2017/papers/thpab020.pdf.

[6] M. Migliorati and M. Zobov, Impedance and instabilities in lepton colliders, in International Workshop on Impedances and Beam Instabilities in Particle Accelerators, Benevento, Italy, 2017, https://agenda.infn.it/contributionDisplay.py? contribId $=101 \&$ confId $=12603$.

[7] N. Nakamura, Effects of longitudinal and transverse resistive-wall wakefields on ERLS, in Proceedings of ERLO9, Ithaca, New York, USA (2009), p. 85, http://accelconf.web .cern.ch/AccelConf/ERL2009/papers/js205.pdf.
[8] K. Shibata, H. Hisamatsu, K. Kanazawa, Y. Suetsugu, and M. Shirai, Development of TiN coating system for beam ducts of KEK B-factory, in Proceedings of the 11th European Particle Accelerator Conference, Genoa, 2008 (EPS-AG, Genoa, Italy, 2008), p. 1700.

[9] A. Rossi, SEY and electron cloud build-up with NEG materials, in 31st Advanced ICFA Beam Dynamics Workshop on Electron-Cloud Effects, Napa, CA, USA (2004), p. 113, DOI: 10.5170/CERN-2005-001.113.

[10] C. Yin Vallgren, G. Arduini, J. Bauche, S. Calatroni, P. Chiggiato, K. Cornelis, P. Costa Pinto, E. Metral, G. Rumolo, E. Shaposhnikova, M. Taborelli, and G. Vandoni, Amorphous carbon coatings for mitigation of electron cloud in the CERN SPS, in Proceedings of the International Particle Accelerator Conference, Kyoto, Japan (ICR, Kyoto, 2010), p. 2033.

[11] L. Palumbo, V. G. Vaccaro, and M. Zobov, Wake fields and impedance, CERN Report No. CERN 95-06, 1995.

[12] D. Shatilov, Luminosity optimization for FCC-ee: recent results, in 59th FCC-ee Optics Design meeting, CERN, Geneva, Switzerland, (2017), https://indico.cern.ch/event/ 661041/contributions/2697585/attachments/1512904/ 2359918/lumi_param.pdf.

[13] D. Boussard, Observation of microwave longitudinal instabilities in the CPS, CERN Report No. CERN LabII/RF/ 75-2, 1975.

[14] A. W. Chao, Physics of Collective Beam Instabilities in High Energy Accelerators (John Wiley \& Sons, New York, 1993).

[15] T. F. Günzel, Transverse coupling impedance of the storage ring at the European Synchrotron Radiation Facility, Phys. Rev. ST Accel. Beams 9, 114402 (2006).

[16] A. Chao, S. Heifets, and B. Zotter, Tune shifts of bunch trains due to resistive vacuum chambers without circular symmetry, Phys. Rev. ST Accel. Beams 5, 111001 (2002).

[17] A. Blednykh, G. Bassi, Y. Hidaka, V. Smaluk, and G. Stupakov, Low-frequency quadrupole impedance of undulators and wigglers, Phys. Rev. Accel. Beams 19, 104401 (2016).

[18] Y. Suetsugu,K. Kanazawa, K. Shibata, T. Ishibashi, H. Hisamatsu, M. Shirai, and S. Terui, Design and construction of the SuperKEKB vacuum system, J. Vac. Sci. Technol. A 30, 031602 (2012).

[19] R. Kersevan, Proposal and simulation of the FCC-ee vacuum system, in Proceedings FCC Week 2017-31 May 2017, Berlin (Germany), https://indico.cern.ch/ event/556692/contributions/2487639/.

[20] C. Scheuerlein, B. Henrist, N. Hilleret, and M. Taborelli, The secondary electron yield of TiZr and TiZrV nonevaporable getter thin film coatings, Appl. Surf. Sci. 172, 95 (2001).

[21] N. Wang and Q. Qin, Resistive-wall impedance of two-layer tube, Phys. Rev. ST Accel. Beams, 10, 111003 (2007).

[22] N. Mounet, The LHC transverse coupled-bunch instability, Ph.D. thesis, École Polytechnique Fédérale de Lausanne (EPFL), Lausanne, Switzerland, 2012.

[23] L. Vos, The impedance of multi-layer vacuum chambers, CERN Report No. CERN-AB-2003-093 (ABP), 2003, https://cds.cern.ch/record/685131.

[24] https://github.com/PyCOMPLETE/PyHEADTAIL. 
[25] M. Migliorati and L. Palumbo, Multibunch and multiparticle simulation code with an alternative approach to wakefield effects, Phys. Rev. ST Accel. Beams 18, 031001 (2015).

[26] R. Boni, A. Drago, A. Gallo, A. Ghigo, F. Marcellini, M. Migliorati, F. Sannibale, M. Serio, A. Stella, G. Vignola, and M. Zobov, DAФNE accumulator ring coupling impedance measurements, Nucl. Instrum. Methods Phys. Res., Sect. A 418, 241 (1998); M. Zobov et al., arXiv:physics/0312072.

[27] M. Migliorati, S. Persichelli, H. Damerau, S. Gilardoni, S. Hancock, and L. Palumbo, Beam-wall interaction in the CERN Proton Synchrotron for the LHC upgrade, Phys. Rev. ST Accel. Beams, 16, 031001 (2013).

[28] J. Haïssinski, Exact longitudinal equilibrium distribution of stored electrons in the presence of self-fields, Il Nuovo Cim. Soc. Ital. Fis. 18B, 72 (1973).

[29] A. W. Chao, K. H. Mess, M. Tigner, and F. Zimmermann, Handbook of Accelerator Physics and Engineering (World Scientific, Singapore, 1999).

[30] S. Hansen, H. G. Hereward, A. Hofmann, K. Hübner, and S. Myers, Effects of space charge and reactive wall impedance on bunched beams, IEEE Trans. Nucl. Sci. 22, 1381 (1975).
[31] K. Ohmi, N. Kuroo, K. Oide, D. Zhou, and F. Zimmermann, Coherent Beam-Beam Instability in Collisions with a Large Crossing Angle, Phys. Rev. Lett. 119, 134801 (2017).

[32] D. Shatilov, FCC-ee parameter optimization, ICFA Beam Dyn. Newslett. 72, 30 (2017).

[33] N. Mounet, DELPHI: an analytic Vlasov solver for impedance-driven modes (2014), http://cds.cern.ch/record/ 1954277/files/CERN-ACC-SLIDES-2014-0066.pdf.

[34] S. White, X. Buffat, N. Mounet, and T. Pieloni, Transverse mode coupling instability of colliding beams, Phys. Rev. ST Accel. Beams 17, 041002 (2014).

[35] K. Y. Ng, Physics of Intensity Dependent Beam Instabilities (World Scientific, Singapore, 2005), ch. 11.

[36] See, as example, A.Drago et al., High current multibunch operation at DAФNE, Proceedings of the Particle Accelerator Conference, Chicago, IL, 2001 (IEEE, New York, 2001), p. 3543-3545.

[37] A. Drago, Feedback systems for FCC-ee, in Proceedings of eeFACT2016, Cockcroft Institute, Daresbury, UK (2016), p. 136-142, TUT3AH9, http://accelconf.web.cern.ch/ AccelConf/eefact2016/papers/tut3ah9.pdf. 